\title{
WAVE and CalFit - Towards Social Interaction in Mobile Body Sensor Networks
}

\author{
Posu Yan ${ }^{\dagger}$, Irving Lin ${ }^{\dagger}$, Michael Roy ${ }^{\dagger}$, Edmund Seto ${ }^{\ddagger}$, Curtis Wang ${ }^{\dagger}$, Ruzena Bajcsy ${ }^{\dagger}$ \\ $\dagger$ Electrical Engineering and Computer Sciences, University of California, Berkeley; CA 94720, USA \\ $\ddagger$ School of Public Health, University of California, Berkeley; CA 94720, USA
}

\begin{abstract}
WAVE is an API for Android OS which which allows for easy management of body sensor networks (BSNs) on mobile platforms. It presents a simple framework for health-oriented applications by providing functionality for data collection from sensors and data processing. CalFit is an interactive application built using WAVE that leverages the power of social influence to promote physical activity.
\end{abstract}

\section{INTRODUCTION}

The emergence of small wireless sensors over the past few years has given rise to many body sensor network (BSN) systems, such as CodeBlue [4], and DexterNet [2], to name a couple. Typically these research-oriented systems are geared towards monitoring people's biological states and actions using the latest in hardware (i.e. proprietary sensors) and software.

The increasing popularity of BSNs has also brought about the rise of consumer-oriented systems, which differ greatly in their scope and goals. Commercial products such as the Nike+ and Nintendo's Wii Fit do not necessarily present advanced systems, but are able to achieve their goals of promoting fitness and exercise due to social cooperation and competition [3].

In this paper, we present WAVE and CalFit, whose aim is to fuse the cutting-edge BSN technology of recent research-oriented health systems with the social framework and simplicity of commercial systems. WAVE is an API for the Android mobile operating system and CalFit is a WAVE application designed to showcase the effectiveness of social interaction in mobile BSNs.

\section{WAVE}

WAVE is an API for the Android OS that allows for quick BSN application development on mobile platforms. This includes providing simple methods for setting up sensors, automatic intra-BSN communication, access to powerful data processing algorithms, and a database interface for storing and accessing data. WAVE provides a simple application interface to BSNs through

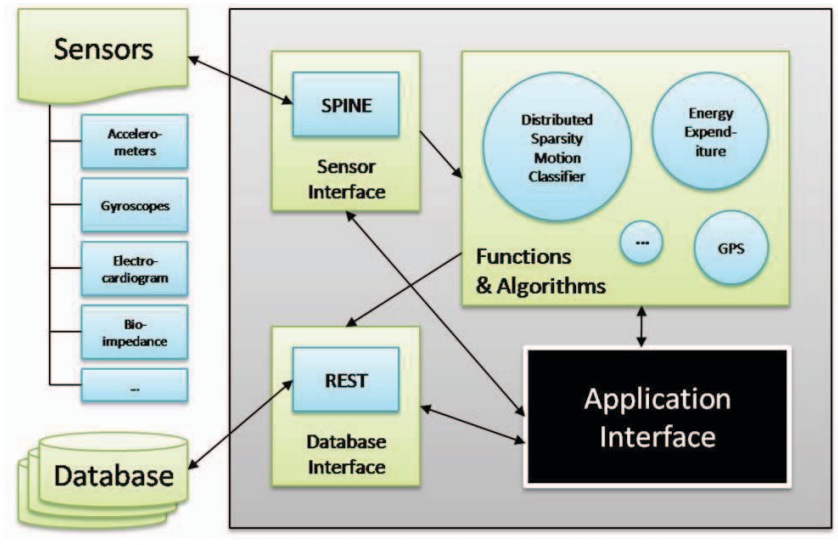

Fig. 1. Architecture of WAVE.

its sensor interface, database interface, and common data analysis algorithms (Fig. 1).

\section{A. Intra-BSN Communication}

WAVE handles intra-BSN communication through the use of the SPINE (Signal Processing In Node Environment) [5] framework. SPINE provides automatic discovery and configuration of sensor nodes, and handles local signal processing capabilities on each node.

SPINE also implements a low-level protocol of communication between nodes, which provides a common interface for all nodes and allows for easy integration of new sensors. With such a protocol, users of WAVE can quickly develop BSN applications without the need to deal with low-level intra-BSN communication.

\section{B. Database Interface}

WAVE provides a simple interface to store and access data in databases. By using REST architecture, a developer can easily setup a database for a WAVE application through a common REST interface. 


\section{Data Processing}

WAVE aims to simplify data processing by providing simple interfaces to commonly used algorithms. In particular, it provides 1) energy expenditure estimation 2) action classification 3) GPS functions.

1) Energy Expenditure Estimation: Using the builtin accelerometer found on many mobile devices, WAVE provides an energy expenditure estimation algorithm designed for a single tri-axial accelerometer [1]. The algorithm calculates a kilocalorie value at a user-specified interval while the application is running, providing the user with real-time statistics.

2) Action Classification: The WAVE API features a distributed sparsity activity classifier [6], allowing developers to easily use action recognition in their applications without the need to implement complicated algorithms. In CalFit, user actions such as running, walking, and jumping, are classified as such by processing the sensor data. With these classifications, more detailed information can be provided as feedback to the user and more accurate data processing (such as caloric expenditure) can be performed. Users of CalFit will be able to see a summary of their progress, including statistics of specific actions (running, walking, jumping, etc.).

The action classification functions in WAVE have been optimized for mobile devices due to their relative lack of computing power. This entails approximating floating point operations with integer operations, as well as tradeoffs between accuracy and performance.

3) GPS: The WAVE API also facilitates the utilization of location data, providing a wrapper for the Google Maps API. WAVE includes methods for estimating the speed, direction, and distance of a user's trajectory, removing the complication of manual GPS configuration as currently present in the Android API. The CalFit Application uses WAVE to provide the user with a map of the current area, along with a highlighted route.

\section{CALFIT}

CalFit is a multi-user application built using WAVE that monitors daily physical activity and encourages exercise through social interaction and competition. It utilizes the energy expenditure estimation and GPS functions of WAVE to collect data on a user's activity, which is then sent to a database containing all user statistics. These statistics are used to generate rankings of each person's performance which are fed back to the user, creating a competitive and social environment. The goal of CalFit is to leverage social interaction and competition to encourage a more active lifestyle.

\section{A. Feedback And Social Interaction}

CalFit periodically calculates and uploads the user's caloric expenditure to a central database. A ranking of users is generated based on this metric and is the primary motivating factor in encouraging more physical activity. Users will also be able to join exercise teams, which fosters both intra-team motivation and inter-team competition, allowing users to be exposed to encouragement from teammates as well as social pressure from competition.

\section{B. Health Experiments}

We plan to study the efficacy of CalFit for a cohort of 30 college undergraduate students. The study will assess differences in physical activity levels between an experimental group with social networking enabled and a control group without social networking enabled. If these initial studies produce encouraging results, an expanded evaluation of CalFit for varying age and socioeconomic statuses would be warranted.

\section{FUTURE WORK}

Currently WAVE and CalFit are works-in-progress. As the Android Bluetooth API has recently been released, we are working to incorporate Bluetooth communication with external sensors. However, we are looking into alternatives to enable Bluetooth functionality. Additionally, we would like to incorporate more varied social networking paradigms, such as interfacing with social networking websites and the ability for a user to search for competitors by proximity. Finally, we intend to port WAVE to other popular mobile platforms.

\section{REFERENCES}

[1] K. Chen and M. Sun. Improving energy expenditure estimation by using a triaxial accelerometer. Journal of Applied Physiology, 83:21122122, 1997.

[2] P. Kuryloski, A. Giani, R. Giannantonio, K. Gilani, R. Gravina, V. Sepp, et al. DexterNet: An Open Platform for Heterogeneous Body Sensor Networks and Its Applications. BSN 2009, 2009.

[3] Lin, J., Mamykina L., Lindtner, S., Delajoux, G. and Strub, H. Fish'n'Steps: Encouraging Physical Activity with an Interactive Computer Game. UbiComp 2006: Ubiquitous Computing (2006), pp. 261-278.

[4] D. Malan, T. Fulford-Jones, M. Welsh, and S. Moulton. CodeBlue: An ad hoc sensor network infrastructure for emergency medical care. In Workshop on Wearable and Implantable Body Sensor Networks, 2004.

[5] T. S. Team. The spine manual version 1.2. Technical report, Telecom Italia Lab, 2008.

[6] Allen Yang, Roozbeh Jafari, Shankar Sastry, and Ruzena Bajcsy. Distributed Recognition of Human Actions Using Wearable Motion Sensor Networks. Journal of Ambient Intelligence and Sensor Environments (JAISE), 2009. 